\title{
4 Graph Theory Applied to Speech: Insights on Cognitive Deficit Diagnosis and Dream Research
}

\author{
Natália Bezerra Mota, Mauro Copelli, and \\ Sidarta Ribeiro
}

\begin{abstract}
In the past ten years, graph theory has been widely employed in the study of natural and technological phenomena. The representation of the relationships among the units of a network allow for a quantitative analysis of its overall structure, beyond what can be understood by considering only a few units. Here we discuss the application of graph theory to psychiatric diagnosis of psychoses and dementias. The aim is to quantify the flow of thoughts of psychiatric patients, as expressed by verbal reports of dream or waking events. This flow of thoughts is hard to measure but is at the roots of psychiatry as well as psychoanalysis. To this end, speech graphs were initially designed with nodes representing lexemes and edges representing the temporal sequence between consecutive words, leading to directed multigraphs. In a subsequent study, individual words were considered as nodes and their temporal sequence as edges; this simplification allowed for the automatization of the process, effected by the free software SpeechGraphs. Using this approach, one can calculate local and global attributes that characterize the network structure, such as the total number of nodes and edges, the number of nodes present in the largest connected and the largest strongly connected components, measures of recurrence such as loops of 1, 2, and 3 nodes, parallel and repeated edges, and global measures such as the average degree, density, diameter, average shortest path, and clustering coefficient. Using these network attributes we were able to automatically sort schizophrenia and bipolar patients undergoing psychosis, and also to separate these psychotic patients from subjects without psychosis, with more than $90 \%$ sensitivity and specificity. In addition to the use of the method for strictly clinical purposes, we found that differences in the content of the verbal reports correspond to structural differences at the graph level. When reporting a dream, healthy subjects without psychosis and psychotic subjects with bipolar disorder produced more complex
\end{abstract}


graphs than when reporting waking activities of the previous day; this difference was not observed in psychotic subjects with schizophrenia, which produced equally poor reports irrespective of the content. As a consequence, graphs of dream reports were more efficient for the differential diagnosis of psychosis than graphs of daily reports. Based on these results we can conclude that graphs from dream reports are more informative about mental states, echoing the psychoanalytic notion that dreams are a privileged window into thought. Overall these results highlight the potential use of this graph-theoretical method as an auxiliary tool in the psychiatric clinic. We also describe an application of the method to characterize cognitive deficits in dementia. In this regards, the SpeechGraph tools were able to sensitize a neuropsychological test widely used to characterize semantic memory, the verbal fluency test. Subjects diagnosed with Alzheimer's dementia were compared to subjects diagnosed with moderate cognitive impairment, either with amnestic symptoms only or with damage in multiple domains. Also studied were elderly individuals with no signs of dementia. The subjects were asked to report as many names of different animals as they could remember within one minute. The sequence of animal names was represented as a word graph. We found that subjects with Alzheimer's dementia produced graphs with fewer words and elements (nodes and edges), higher density, more loops of three nodes, and smaller distances (diameter and average shortest path) than subjects in the other groups; a similar trend was observed for subjects with moderate cognitive impairment, in comparison to elderly adults without dementia. Furthermore, subjects with moderate cognitive impairment with amnestic deficits only produced graphs more similar to the elderly without dementia, while those with impairments in multiple domains produced graphs more similar to the graphs from individuals with Alzheimer's dementia. Importantly, also in this case it was possible to automatically classify the different diagnoses only using graph attributes. We conclude by discussing the implications of the results, as well as some questions that remain open and the ongoing research to answer them.

\subsection{Introduction}

Every day when we wake up, before talking with other people, we talk with ourselves using inner speech to remember what day it is, where we are, to make plans about what to do in the next few minutes or hours, who we are going to meet, or what we are supposed to do. When we recognize this "inner speech" 
as coming from ourselves, we may simply call it "thinking." However, sometimes this inner speech is not recognized as self, but rather as stimuli generated elsewhere; this is the basis of what we call psychosis. Sometimes past memories dominate this mental space, and we focus on past feelings of sadness, joy, fear, or anxiety. Past and future memories are mixed in these first moments even before any interaction with another person. This flow of memories and thoughts helps organize our actions and to soothe our anxiety and sadness, as we can plan future solutions to solve past problems. Organized, healthy mental activity allows old and new information to interact in order to support different actions that take experience into account in an integrated manner. But what happens with this flow of thoughts when we are unable to organize our inner space?

For centuries, psychiatry has described symptoms known as thought disorder that reflects disorganization of this flow of ideas, memories, and thoughts (Andreasen \& Grove, 1986; Kaplan \& Sadock, 2009). Those symptoms are related with psychosis, a syndrome characterized by hallucinations (when one perceives an object that does not exist; a sensorial perception without a real external object) and delusions (when one believes in realities that do not exist for other people; ideas or beliefs not real for their peers) (Kaplan \& Sadock, 2009). There are many different causes for psychosis, such as the use of psychoactive substances or neurological conditions such as cerebral tumors or epilepsies. However, psychotic symptoms may occur without a clear cause, starting with a strange feeling or perception, getting worse, creating a confused reality hard to share even with the closest person, and causing major mental suffering.

In association with this strange reality, the patient can experience the feeling of fragmentation of thoughts, having difficulty to organize ideas or to follow a flow of memories, impacting the way to express what they are thinking or feeling, creating meaningless speech (symptoms known as "alogia," and "poor speech"). This frequently reflects a mental disorder known as schizophrenia. In other cases, the person may experience another aberrant organization of thought, with higher speed of mental activity, associating different memories and ideas (known as "flight of thoughts"), creating a speech with large amount of words (a symptom known as "logorrhea") that never reaches the main point. This pattern of thought disorder is common during the mania phase of bipolar disorder, a psychiatric condition mainly described by opposite mood cycles comprising depressive and manic phases. This speech pattern changes during depressive phases in the opposite direction (low speed of thought, fewer associations, fewer amount of words during speech). The speech content can reflect that strange psychotic reality on all those conditions with unlikely word association, but the organization of ideas reflected in the word trajectories reveals different directions of thought disorder, helping psychiatrists make differential 
diagnosis between bipolar disorder and schizophrenia, predicting different life courses and cognitive impacts.

The description of these different patterns of thought organization perceived through language helped psychiatrists distinguish between two different pathological states and predict different life courses (with higher cognitive deficits for schizophrenia, first known as Dementia Precox [Bleuler, 1911]). However, recognizing these features subjectively requires a long-term professional training and adequate time with each patient to know each individual and avoid misjudgments. And even with the best evaluation conditions it is only possible to quantify those features subjectively, judging disease severity by grades on the psychometric scales such as BPRS and PANSS (Bech, Kastrup, \& Rafaelsen, 1986; Kay, Fiszbein, \& Opler, 1987). The differential diagnosis requires at least six months of observation during the first episode (First, Spitzer, Gibbon, \& Williams, 1990), which means that the initial treatment may occur under considerable doubt regarding the diagnostic hypothesis. This lack of objective quantitative evaluation also negatively impacts the research strategies that aim to find biomarkers for complex psychiatric conditions (Insel, 2010).

Another condition that benefits from early diagnosis and correct interventions to prevent major cognitive damage is Alzheimer's Disease (AD) (Daviglus et al., 2010; Kaplan \& Sadock, 2009; Riedel, 2014). Specific characterization of risk during preclinical $\mathrm{AD}$ requires specialized investigations and still challenges professionals in the field, due to a lack of a consensual description of each stage (Daviglus et al., 2010; Riedel, 2014). Failure to recognize AD early on can lead to a loss of opportunity to prevent cognitive decline (Daviglus et al., 2010; Riedel, 2014). In summary, the currently poor quantitative characterization of cognitive impairments related to pathological conditions such as psychosis or dementia hinders the early detection of these conditions. In this scenario, the new field of computational psychiatry has been proposing mathematical tools to better quantify behavior (Adams, Huys, \& Roiser, 2015; Montague, Dolan, Friston, \& Dayan, 2012; Wang, \& Krystal, 2014).

To this end, natural language processing tools are particularly interesting. It is now possible to simulate the expert's subjective evaluation with better precision and reliability, either by quantifying specific content features such as semantic incoherence (Bedi et al., 2015; Cabana, Valle-Lisboa, Elvevag, \& Mizraji, 2011; Elvevåg, Foltz, Weinberger, \& Goldberg, 2007), or by analyzing the structural organization of word trajectories recorded from patients (Bertola et al., 2014; Mota et al., 2012; Mota et al., 2014).

\subsection{Semantic Analysis for the Diagnosis of Psychosis}

One useful tool used to characterize the incoherent speech characteristic of psychotic crises is called Latent Semantic Analysis (LSA) (Landauer \& Dumais, 
1997). The strange reality created during psychotic states impacts the coherence of the flow of words when patients express their thoughts freely, leading to improbable connections between semantically distant words within the same sentences.

LSA is based on a model that assumes that the meaning of each word is a function of its relationship with the other words in the lexicon (Landauer \& Dumais, 1997). By this rationale, if two words are semantically similar, i.e., if their meanings are related, they must co-occur frequently in texts. It follows that if one has a large enough database of word co-occurrences in a large enough corpus of texts, it is possible to represent each word of that corpus as a vector in a semantic space, and their proximity in that space will be interpreted as semantic similarity (Landauer \& Dumais, 1997).

When healthy subjects describe their normal reality, it is expected that they will use words that are semantically similar within the same text. However, when reality becomes bizarre, as typical of psychotic states, subjects are expected to use semantically distant words in sequence, thus building incoherent speech. That incoherence can be quantified as a measure of semantic distance between consecutive words or sets of words (for example, a set of words used in the same sentence). The more incoherent the speech, the larger the semantic distance between consecutive words or set of words. This was first shown for chronic patients with schizophrenia diagnosis (Elvevåg et al., 2007) and helped predict diagnosis in the prodrome phase, 2.5 years before the first psychotic crises (Bedi et al., 2015).

\subsection{What Is a Speech Graph?}

One way to quantify thought disorder is to represent the flow of ideas and memories reflected on the flow of words during a free speech as a trajectory and create a speech graph. A graph is a set of nodes linked by edges (formally defined as $\mathrm{G}=(\mathrm{N}, \mathrm{E})$, being $\mathrm{N}=\{\mathrm{w} 1, \mathrm{w} 2, \ldots, \mathrm{wn}\}$ and $\mathrm{E}=\{(\mathrm{wi}, \mathrm{wj})\}$ [Bollobas, 1998; Börner, Sanyal, \& Vespignani, 2007]). The criteria determining how a link is established between two nodes define topological properties of these graphs that can be measured locally or globally. In the present case, each word is defined as a node, and the temporal sequence of words during a free speech is represented by directed edges (Mota et al., 2014) (Figure 4.1). From a speech graph we can objectively measure local and global features of the word trajectory that reflects the flow of thoughts during a free speech task (like when the subject reports a daily event, a past memory, or even a dream memory).

In the last decade, graph theory has been widely employed in the study of natural or technological phenomena (Boccaletti et al., 2006). By allowing the representation of the relationships among their units, the overall structure of a network can elucidate characteristics that could not be understood by 


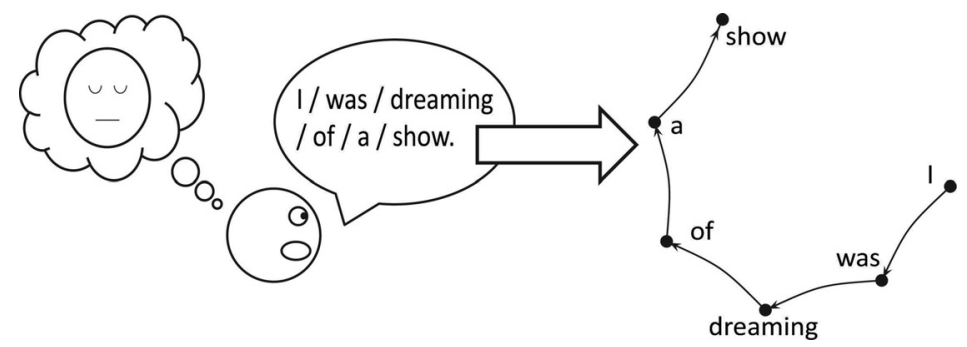

Figure 4.1 Examples of speech graphs from dream reports of schizophrenic, bipolar, and control subjects. Starting from transcribed verbal reports, graphs were generated using custom-made Java software (see the following text). Figure from Mota et al. (2014).

considering only a few units. The meaning of the represented structure basically depends on what is being considered as a node and on the definition of the presence and direction of edges (links between nodes). Graph theory as a tool not only may help tackle problems in the basic sciences but can also be applied to solve complex problems in everyday life, otherwise difficult to characterize and measure. An interesting strategy in scientific research is to keep both goals in focus: seek to understand a phenomenon at the fundamental level, while at the same time use the knowledge as a tool to solve practical problems (Stokes, 1997). With a simultaneous focus on basic and applied research, the application of graph theory to represent the relationship between spoken words helps understand how different psychiatric conditions differentially impact the flow of words during free speech, and how we can apply this knowledge to perform differential diagnosis.

Once reports are represented as graphs, one can calculate several attributes that quantify local and global characteristics. We calculated 14 attributes comprising 2 general graph attributes (Nodes and Edges), 5 recurrence attributes (Parallels - PE and Repeated Edges - RE; Loops of one - L1, two - L2 and three nodes - L3), 2 attributes of connectivity (Largest Connected Component - LCC and Largest Strongly Connected Component - LSC) and 5 global attributes (Average Total Degree - ATD, Density, Diameter, Average Shortest Path - ASP, Clustering Coefficient - CC) (Figure 4.2).

In order to compare graphs with different number of elements (controlling verbosity difference as measured by different amounts of words), two main strategies were used. First we divided each graph attribute by the amount of words in the report, assuming a linear relationship between graph attribute and verbosity. A pertinent critique is that the relationship between graph attributes and verbosity is not always linear, and for some attributes it is not clear if there is a direct relationship (Figure 4.3). A second strategy was to attribute a graph for 


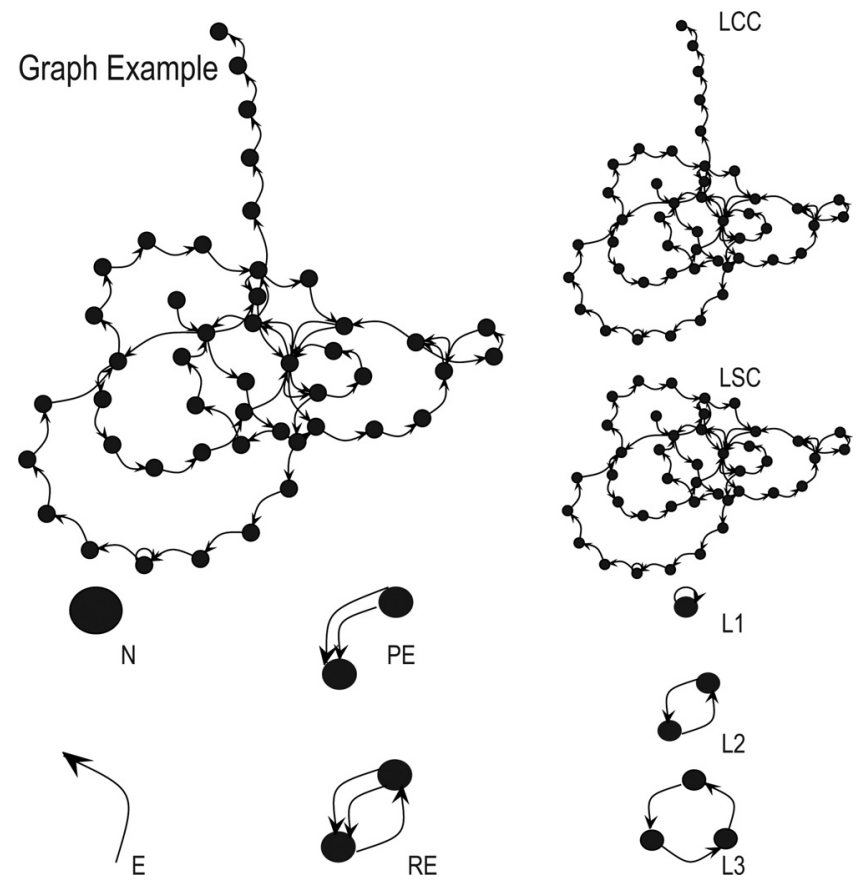

Figure 4.2 Examples of Speech Graph Attributes described earlier (figure from Mota et al., 2014).

\section{Speech Graph Attributes:}

1. N: Number of nodes.

2. E: Number of edges.

3. RE (Repeated Edges): sum of all edges linking the same pair of nodes.

4. PE (Parallel Edges): sum of all parallel edges linking the same pair of nodes given that the source node of an edge is the target node of the parallel edge.

5. L1 (Loop of one node): sum of all edges linking a node with itself, calculated as the trace of the adjacency matrix.

6. L2 (Loop of two nodes): sum of all loops containing two nodes, calculated by the trace of the squared adjacency matrix divided by two.

7. L3 (Loop of three nodes): sum of all loops containing three nodes (triangles), calculated by the trace of the cubed adjacency matrix divided by three.

8. LCC (Largest Connected Component): number of nodes in the maximal subgraph in which all pairs of nodes are reachable from one another in the underlying undirected subgraph. When you have all the words on one large connected component, LCC will be the same as N.

(continued) 
each set of a fixed number of words, skipping an also fixed number of words to build the next graph, assuming a certain level of overlap between consecutive graphs. This "sliding window" approach allows calculating the average graph attributes of a graph with a fixed number of words. This enables the study of topological characteristics of graphs with different reports size (say, small, medium, and big graphs). A critique for this strategy is the arbitrary cut of word sequences that can change topological properties, mainly global attributes. This is an important discussion of ongoing research that needs to be addressed carefully, so as to enable a better interpretation of the results.

\subsection{Speech Graphs as a Strategy to Quantify Symptoms on Psychosis}

In an attempt to represent the flow of thoughts presented in a free speech, speech graphs were initially designed with nodes representing lexemes (a subject, object, or verb on the sentence), and their temporal sequence represented as directed edges, yielding directed multigraphs with self-loops and parallel edges (Mota et al., 2012). Analyzing dream reports represented as graphs from 24 subjects (8 subjects presenting psychotic symptoms with schizophrenia diagnosis, 8 subjects also with psychotic symptoms diagnosed as bipolar disorder in the mania phase and 8 control subjects without any psychotic symptom), it was possible to quantify psychiatric symptoms such as:

(Figure 4.2 caption continued)

9. LSC (Largest Strongly Connected Component): number of nodes in the maximal subgraph in which all pairs of nodes are reachable from one another in the directed subgraph (node a reaches node $b$, and b reaches a).

10. ATD (Average Total Degree): given a node n, its Total Degree is the sum of "in" and "out" edges. Average Total Degree is the sum of Total Degree of all nodes divided by the number of nodes.

11. Density: number of edges divided by possible edges $(\mathrm{D}=2 * \mathrm{E} / \mathrm{N} *(\mathrm{~N}-1))$, where $\mathrm{E}$ is the number of edges and $\mathrm{N}$ is the number of nodes.

12. Diameter: length of the longest shortest path between the node pairs of a network.

13. Average Shortest Path (ASP): average length (number of steps along edges) of the shortest path between pairs of nodes of a network.

14. CC (Average Clustering Coefficient): given a node $n$, the Clustering Coefficient Map (CCMap) is the set of fractions of all $n$ neighbors that are also neighbors of each other. Average $\mathrm{CC}$ is the sum of the Clustering Coefficients of all nodes in the CCMap divided by the number of elements in the CCMap. 

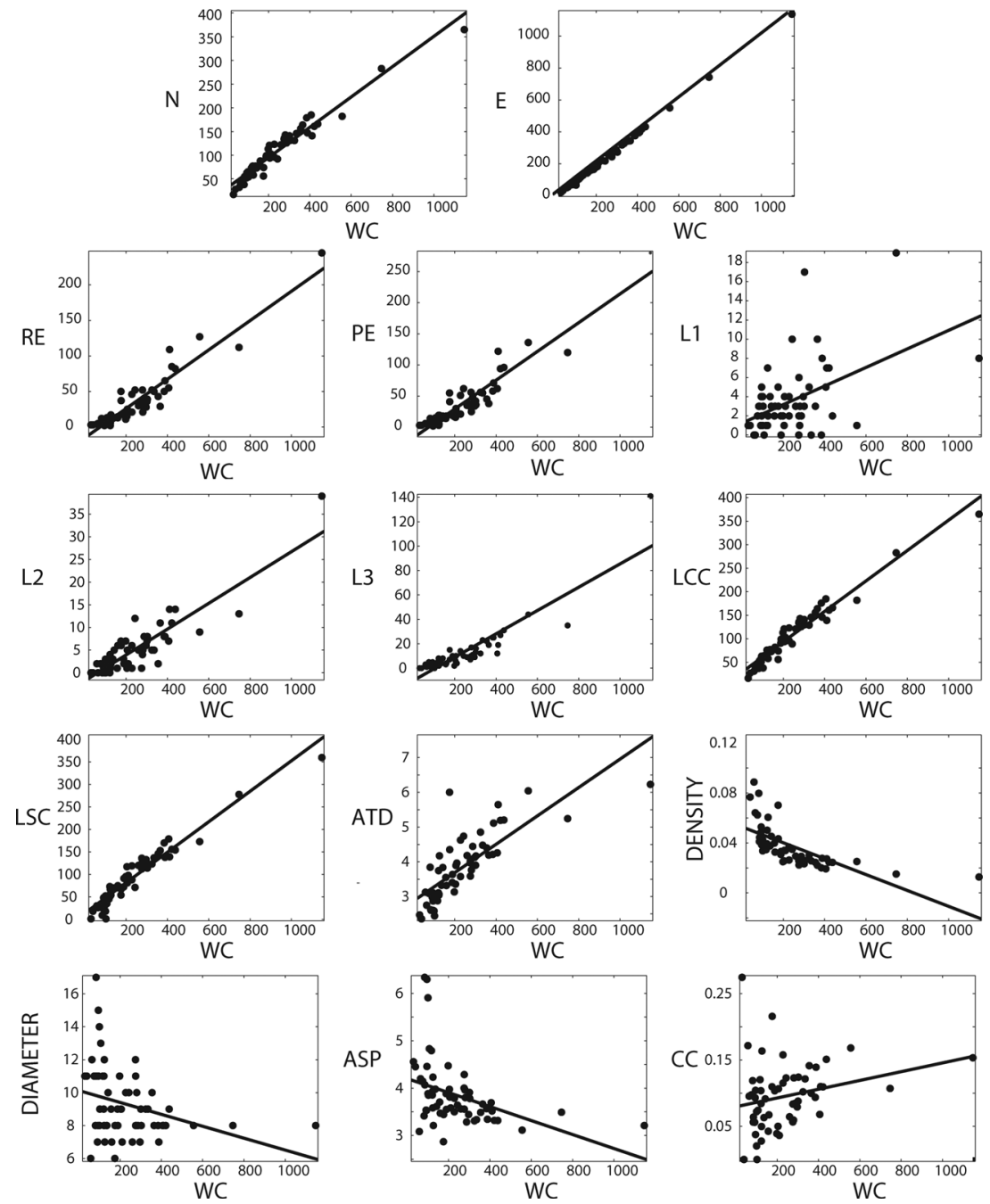

Figure 4.3 Linear correlation between SGA and word count (WC) (figure from Mota et al., 2014).

1. Logorrhea, described as the increase of verbosity characteristic of bipolar disorder on mania phase. This was quantified not only by counting more words in the bipolar group but also by more frequent recurrence (more parallel edges), even when controlling for differences in verbosity by dividing graph attributes by the amount of words in the speech. This means that the reports tend to return more often to the same topics. 
2. Flight of thoughts, described as talking about other topics than the main topic asked, which is also characteristic of bipolar disorder. In the bipolar group, more nodes were used to talk about waking events upon request to report on a recent dream.

3. Poor speech, described as loss of meaning on the speech and perceived as a set of words that are poorly connected, characteristic of schizophrenia. This was quantified as more nodes per words, denoting reports that address the topics only once, neither branching nor recurring, so almost all the words used will count as a different node.

It was possible to automatically sort schizophrenia from bipolar group using a machine learning approach. A Naïve Bayes classifier was used to distinguish between both groups, and to distinguish between pathological groups and nonpsychotic subjects (Kotsiantis, 2007). The classifier received as input either speech graph attributes or grades given from psychiatrists concerning psychiatric symptoms (using standard psychometric scales: PANSS [Kay et al., 1987] and BPRS [Bech et al., 1986]). Classification accuracy was assessed through the calculation of sensitivity, specificity, kappa statistics, and the area under the receiver operating characteristic curve (AUC), described as a plot of sensitivity (or true positive rate) on the $y$-axis versus false positive rate (or 1specificity) on the x-axis. An AUC around 0.5 means a random classification, whereas $\mathrm{AUC}=1$ means a perfect classification (none of the possible errors were made). It was possible to classify the pathological groups against nonpsychotic group using graph attributes and psychometric scales with high accuracy (AUC higher than 0.8) (Table 4.1). But to distinguish between schizophrenia and bipolar groups, graph attributes performed better than psychometric scales (AUC $=0.88$ using graph attributes as input, while $\mathrm{AUC}=0.57$ when using psychometric scales as input) (Table 4.1).

This first study had some limitations concerning the low sample (only eight subjects per group) and the methodology. First, the transformation from a text to a graph was handmade, a process that is time consuming and has a higher risk of error. Second, the graph was not completely free of subject evaluation (a node

Table 4.1 Classification metrics between diagnostic groups using SpeechGraph Attributes (Mota et al., 2012).

\begin{tabular}{lcccc}
\hline \hline & Sensitivity & Specificity & Kappa & AUC \\
\hline $\mathrm{S} \times \mathrm{B}$ & $93.8 \%$ & $93.7 \%$ & 0.88 & 0.88 \\
$\mathrm{~S} \times \mathrm{C}$ & $87.5 \%$ & $87.5 \%$ & 0.75 & 0.90 \\
$\mathrm{~B} \times \mathrm{C}$ & $68.8 \%$ & $68.7 \%$ & 0.37 & 0.80 \\
\hline \hline
\end{tabular}




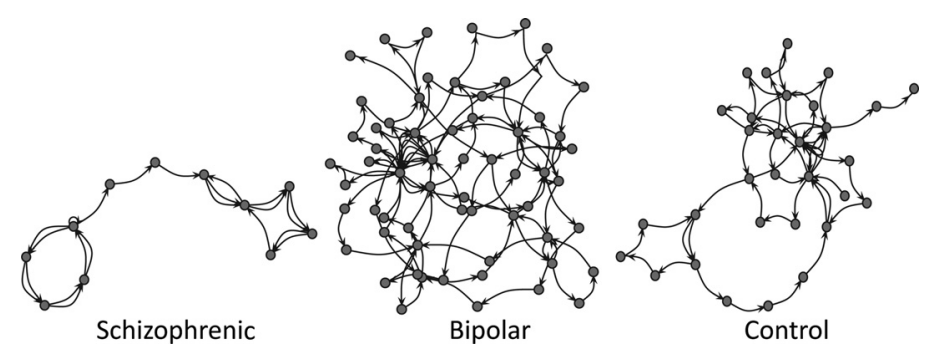

Figure 4.4 Representative speech graphs extracted from dream reports from a schizophrenic, a bipolar and a control subject (figure from Mota et al., 2014).

was considered as a subject, object, or verb on the sentence and, at a grammar level, it required a syntactic evaluation). So, in order to avoid these problems and to allow the study of a larger sample with larger texts, in a subsequent study we employed words as nodes and their temporal sequence as edges, a simplification that allowed the process to be automatized by the SpeechGraphs software (Mota et al., 2014). This custom-made Java software, available at http:// neuro.ufrn.br/softwares/speechgraphs, receives as input a text file and returns the graph based on the text with all the 14 graph attributes described before. It is also possible to cut the text in consecutive graphs with a fixed number of words, controlling for verbosity and exploring different sizes of word windows to study cognitive phenomena.

To characterize distinct pathological phenomena in the speech of different types of psychosis, the SpeechGraphs tool was applied. Symptoms of Bipolar Disorder such as logorrhea could still be associated to the increase of the network size (Figure 4.4) (Mota et al., 2014; Mota et al., 2012). Also, symptoms of schizophrenia such as alogia and poor speech were measured as fewer edges (E), and smaller connected components (LCC) and strongly connected components (LSC) when compared to bipolar and control groups (Figure 4.4), producing less complex graphs in the schizophrenia group even after controlling for word count (comparing consecutive graphs of 10, 20, and 30 words with one word as step). In graphs from this group there are fewer edges between nodes and fewer nodes connected by some path or mutually reachable. This means that the schizophrenia group tends to talk only a few times about the same topic, not returning or associating past topics with consecutive ones, probably denoting cognitive deficits such as working memory deficits.

Using these network characteristics it was also possible to automatically sort the schizophrenia and bipolar groups, and those from subjects without psychosis, with AUC $=0.94$ to classify schizophrenia and control groups, $\mathrm{AUC}=0.72$ to classify bipolar and control group, and $\mathrm{AUC}=0.77$ to classify schizophrenia and bipolar groups (Table 4.2). These results 
Table 4.2 Classification metrics between

diagnostic groups using SpeechGraph

Attributes (Mota et al., 2014).

\begin{tabular}{lccc}
\hline \hline & AUC & Sensitivity & Specificity \\
\hline $\mathrm{S} \times \mathrm{B} \times \mathrm{C}$ & 0.77 & 0.62 & 0.81 \\
$\mathrm{~S} \times \mathrm{B}$ & 0.77 & 0.69 & 0.68 \\
$\mathrm{~S} \times \mathrm{C}$ & 0.94 & 0.85 & 0.85 \\
$\mathrm{~B} \times \mathrm{C}$ & 0.72 & 0.74 & 0.75 \\
\hline \hline
\end{tabular}

highlight the potential use of this method as an auxiliary tool in the psychiatric clinic.

To better understand the relationship between these graph features and the symptomatology measured by psychometric scales, the correlation between those metrics was analyzed. Edges, LCC, and LSC were strongly negatively correlated with cognitive and negative symptoms (as measured by psychometric scales). In other words, when the subjects presented more severity on symptoms such as emotional retraction and flattened affect (loss of emotional reaction), poor eye contact (with the interviewer during psychiatric evaluation), loss of spontaneity or fluency on speech, and difficulty in abstract thinking (measured by the ability to interpret proverbs), their reported dreams generated graphs with fewer edges and fewer nodes on the largest connected and strongly connected component. Those psychiatric symptoms are more common in subjects with schizophrenia (Kaplan \& Sadock, 2009), indicating how we can measure the impact on cognition and deficits in social interactions of these individuals through graphs of speech (Mota et al., 2014). Cognitive and psychological aspects that drive this pattern of speech such, as working memory, planning, and theory of mind abilities, may explain those deficits and help elucidate the pathophysiology of the different psychotic disorders. When the interviewer asks the subject to report a memory, the way the subject interacts socially with the interviewer and recalls what to report, planning the answer and the sequence of events to report, impacts the sequence of words spoken, reflecting their mental organization.

\subsection{Differences in Speech Graphs due to Content (waking $\times$ dream reports)}

We already understand that during pathological cognitive states there is an impact on the flow of thoughts or memories that we can track by the word trajectory. But what happens with physiologically altered consciousness states like dream mentation? Is it possible to characterize differences between dream 

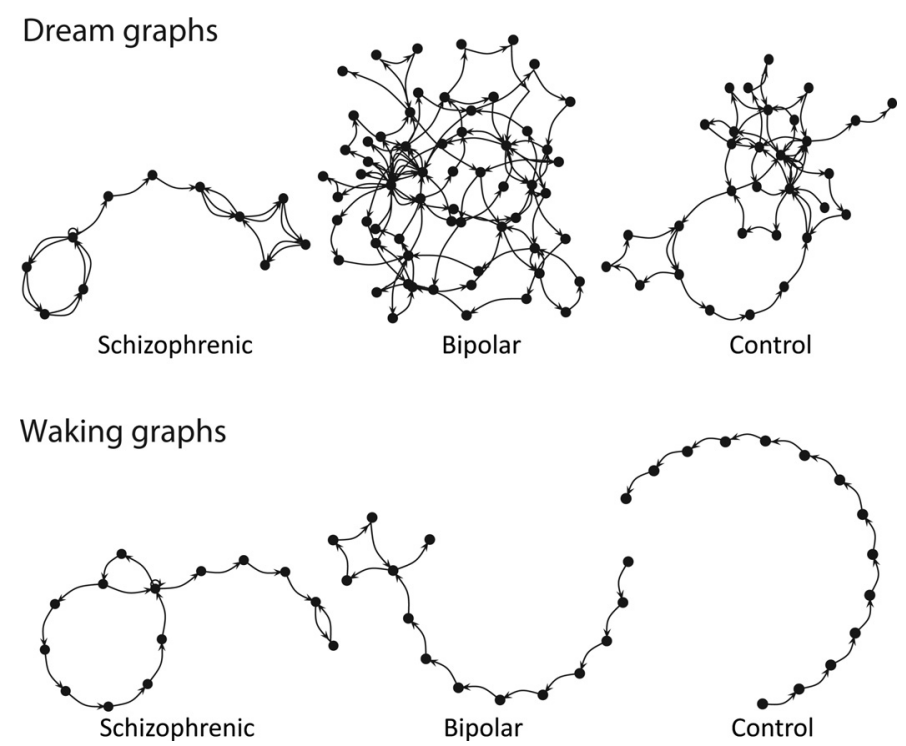

Figure 4.5 Representative speech graphs examples extracted from dream and waking reports from the same schizophrenic, bipolar, and control subject (figure from Mota et al., 2014).

and daily memories regarding word trajectories? Does it inform any additional features about general cognition?

A few minutes before waking up every day we can experiment an exclusively internal reality not shared with our friends or family: dreaming. This reality is internally built based on a set of memories with different affective valences, with different types of meaning only accessible by the dreamer. This confused mental state is phenomenologically similar to a psychotic state, as there is a lack of insight regarding the bizarreness of this strange reality (Dresler et al., 2015; Mota et al., 2014; Scarone et al., 2007). Thus it would not be surprising to expect that the flow of information regarding dream memories could better reveal thought disorganization characteristic of psychotic states.

During the studies with psychotic populations, there were differences in speech graphs depending on the speech content. When reporting a dream, subjects without psychosis and subjects with bipolar disorder produced more complex graphs (higher connectivity) than when reporting daily activities of the previous day, a difference that was not observed in subjects with schizophrenia (those subjects reported dreams or daily memories with the same few connected graphs) (Figure 4.5) (Mota et al., 2014). Therefore, graphs of dream reports were more efficient in group sorting than graphs of daily reports (Mota et al., 2014). 
Another intriguing result was found in the correlations between speech graph attributes and clinical symptoms measured by psychometric scales PANSS (Kay et al., 1987) and BPRS (Bech et al., 1986). Only dream graphs connectivity attributes were strongly and negatively correlated with negative and cognitive symptoms (as measured by both scales) that are more common in schizophrenia. Waking report graphs showed negative correlations between general psychotic symptoms such as loss of insight (measured by PANSS) and incoherent speech (measured by BPRS) with LCC (also a connectivity attribute) (Mota et al., 2014). This emphasizes that reports of dream memories requires different cognitive functions and empathy abilities than reports of daily memories.

Based on these results we can conclude that graphs from dream reports are more informative about mental states than are graphs representing waking reports. This result echoes the psychoanalytic proposal that dreams are a privileged window into thought (Freud, 1900; Mota et al., 2014). This observation has started a new basic research approach to quantitatively understand what is going on when we remember a dream. The use of electrophysiological approaches (most notably, multichannel electroencephalography) to characterize different sleep stages in the laboratory allows the access to dream mentation by their reports at the same time that we access electrophysiological activity during sleep.

\subsection{Speech Graphs Applied to Dementia}

Considering the characterization of cognitive deficits in conditions such as dementia, the use of tests designed to characterize specific cognitive impacts on memory domain are useful in early evaluation. One example is the Verbal Fluency Test, which consists on verbal recall of different names of a specific category (usually animals) during a fixed time. This was first used to investigate the executive aspects of verbal recall, counting the capacity to produce an adequate quantity of words in a limited condition of recall, not repeating or recalling different categories (Lezak, Howieson, Bigler, \& Tranel, 2012). The individual needs to access semantic memory correctly and to be flexible in order to quickly change the words (using temporal cortex structures), and to store the already mentioned words to avoid repetitions, which requires executive functions such as inhibitory control (using frontal cortex structures) (Henry \& Crawford, 2004).

Different pathologies, such as dementia, can damage the performance on this task. As different structures are involved to correctly answer the task, different kinds of errors can help distinguish between different causes (damage in different locations). Different causes of dementia lead to different symptomatology 
evolutions, which represent different location damages. The characterization of word trajectory with the application of the SpeechGraph tool complements this neuropsychological test (Bertola et al., 2014). A total of 100 individuals 25 subjects diagnosed with Alzheimer's dementia, 50 diagnosed with Moderate Cognitive Impairment ( 25 of them with only amnestic symptoms and the others 25 with damage in multiple domains), and 25 elderly subjects with no signs of dementia - were asked to report as many names of different animals as they could remember in one minute (Nickles, 2001). The sequence of animal names was represented as a word graph.

It was observed that subjects with Alzheimer's dementia produced graphs with fewer words and elements (nodes and edges), higher density, more loops of three nodes and smaller distances (diameter and average shortest path) than did other groups, with the same trend for subjects with moderate cognitive impairment compared to elderly adults without dementia (Bertola et al., 2014). Furthermore, subjects with moderate cognitive impairment with only amnestic deficits produced graphs more similar to the elderly without dementia, while those with impairments in multiple domains produced graphs more similar to the graphs from individuals with Alzheimer's disease. Also in this case, it was possible to automatically classify the different diagnoses only from graph attributes (Bertola et al., 2014). There was also correlation between speech graph attributes and two important standard cognitive assessments widely used on geriatric population, denoting an important correlation between word trajectory on verbal fluency recall and general cognitive status (measured with MMSE - Mini Mental State Exam) and functional performance (measured with the Lawton Instrumental Activities of Daily Living Scale) (Bertola et al., 2014).

On one hand, the more cognitively preserved were the elderly, the more unique nodes were produced on less-dense graphs. On the other hand, the more functionally dependent the individuals were, the less words, nodes, and edges were produced on denser graphs with smaller diameter and average shortest paths (Bertola et al., 2014). Another differential impact was evident for three-node loops, a repetition of the same word with only two words in between (example: "lion," "cat," "dog," "lion"), found in higher frequency in the Alzheimer group compared with MCI and control groups (Bertola et al., 2014). This means an impairment in working memory since the early stages of the Alzheimer's disease (already recognized by other working memory assessments [Huntley \& Howard, 2010]).

These results point to the additional information that the characterization of word trajectory brings to a well-established neuropsychological test. On this application example, as the test has restricted rules, we expect that the subject produces a certain type of graph, and different types of deviations from this expected pattern informs about cognitive impairments. 


\subsection{Future Perspectives}

Word graphs are not the only tool to quantify psychiatric symptoms on speech analysis. As pointed out in the introduction, other approaches aim to quantify semantic similarities between words (Bedi et al., 2015; Elvevåg et al., 2007). The relationship between speech incoherence measured by LSA and speech structure measured by Speech Graphs is not clear yet. Both measures take into account word sequences and word co-occurrences, but with very different approaches (one compares with a semantic model based on a large corpus, and the other uses graph theory to characterize topological features of the speech sample). Understanding both approaches better can improve automated speech analysis for clinical purposes such as diagnosis and prognosis prediction, creating useful follow-up tools in a clinical set.

Other interesting perspective is to combine language analysis with prosody analysis. Semiautomated tools have characterized prosodic deficits related to schizophrenia diagnosis. The patients made more pauses, were slower, and showed less pitch variability and fewer variation in syllable timing, expressing a flat prosody when compared to matched controls (Martínez-Sánchez et al., 2015). The relationship between expressive prosody and language features during free speech can elucidate several cognitive characteristics subjectively perceived by well-trained psychiatrists (Berisha, Wang, LaCross, \& Liss, 2015).

A better understanding of word trajectories in free speech can also be applied in settings other than the psychiatric clinic. As these tools show important correlations with cognitive deficits in psychosis and dementia, could it be useful to characterize cognitive development in a school setting? This kind of approach could help predict cognitive impairment early enough to allow quick intervention, preventing learning disabilities that later on would be harder to manage. This could also help quantitatively characterize cognitive development in a naturalistic manner.

\section{Acknowledgments}

The authors dedicate this chapter to the memory of Raimundo Furtado Neto, who made important contributions to the development of the SpeechGraphs software. This work was supported by Conselho Nacional de Desenvolvimento Científico e Tecnológico (CNPq), grants Universal 480053/2013-8 and Research Productivity 306604/2012-4 and 310712/2014-9; Coordenação de Aperfeiçoamento de Pessoal de Nível Superior (CAPES) Projeto ACERTA; Fundação de Amparo à Ciência e Tecnologia do Estado de Pernambuco (FACEPE); FAPESP Center for Neuromathematics (grant \# 2013/07699-0, S. Paulo Research Foundation FAPESP). 


\section{References}

Adams, R. A., Huys, Q. J., \& Roiser, J. P. (2015). Computational psychiatry: towards a mathematically informed understanding of mental illness. Journal of Neurology, Neurosurgery \& Psychiatry. doi:jnnp-2015-310737.

Andreasen, N. C., \& Grove, W. M. (1986). Thought, language, and communication in schizophrenia: diagnosis and prognosis. Schizophrenia Bulletin, 12(3), 348359.

Bech, P., Kastrup, M., \& Rafaelsen, O. J. (1986). Mini-compendium of rating scales for states of anxiety depression mania schizophrenia with corresponding DSM-III syndromes. Acta Psychiatrica Scandinavica Supplementum, 326, 1-37.

Bedi, G., Carrillo, F., Cecchi, G. A., Slezak, D. F., Sigman, M., Mota, N. B., Ribeiro, S., Javitt, D., Copelli, M., \& Corcoran, C. M. (2015). Automated analysis of free speech predicts psychosis onset in high-risk youths. Nature Partner Journals Schizophrenia, 1, 15030. doi:10.1038/npjschz.2015.30.

Berisha, V., Wang, S., LaCross, A., Liss, J. (2015). Tracking discourse complexity preceding Alzheimer's disease diagnosis: A case study comparing the press conferences of Presidents Ronald Reagan and George Herbert Walker Bush. Journal of Alzheimer's Disease, 45, 3.

Bertola, L., Mota, N. B., Copelli, M., Rivero, T., Diniz, B. S., Romano-Silva, M. A., Ribeiro, S., \& Malloy-Diniz, L. F. (2014). Graph analysis of verbal fluency test discriminate between patients with Alzheimer's disease, mild cognitive impairment and normal elderly controls. Frontiers in Aging Neuroscience, 6, 185. doi:10.3389/fnagi.2014.00185.

Bleuler, E. (1911). Dementia praecox or the group of schizophrenias. (J. Zinkin, Trans.). New York: International Universities Press.

Bollobas, B. (1998). Modern graph theory. Berlin: Springer-Verlag.

Börner, K., Sanyal, S., \& Vespignani, A. (2007). Network science. In B. Cronin (Ed.), Information today (pp. 537-607). Medford, NJ: ARIST.

Cabana, A., Valle-Lisboa, J. C., Elvevag, B., \& Mizraji, E. (2011). Detecting orderdisorder transitions in discourse: Implications for schizophrenia. Schizophrenia Research. doi:S0920-9964(11)00233-7.

Daviglus, M. L., Bell, C. C., Berrettini, W., Bowen, P. E., Connolly, E. S., Jr., Cox, N. J., Dunbar-Jacob, J. M., Granieri, E. C., Hunt, G., McGarry, K., Patel, D., Potosky, A. L., Sanders-Bush, E., Silberberg, D., \& Trevisan, M. (2010). NIH state-ofthe-science conference statement: Preventing Alzheimer's disease and cognitive decline. NIH Consensus and State-of-the-Science Statements, 27(4), 1-30.

Dresler, M., Wehrle, R., Spoormaker, V. I., Steiger, A., Holsboer, F., Czisch, M., \& Hobson, J. A. (2015). Neural correlates of insight in dreaming and psychosis. Sleep Medicine Reviews, 20, 92-99. doi:10.1016/j.smrv.2014.06.004.

Elvevåg, B., Foltz, P. W., Weinberger, D. R., \& Goldberg, T. E. (2007). Quantifying incoherence in speech: An automated methodology and novel application to schizophrenia. Schizophrenia Research, 93(1-3), 304-316.

First, M. H., Spitzer, R. L., Gibbon, M., \& Williams, J. (1990). Structured clinical interview for DSM-IV Axis I Disorders - Research Version, Patient Edition (SCID-I/P). New York: Biometrics Research, New York State Psychiatric Institute.

Freud, S. (1900). The interpretation of dreams. (J. Strachey, Trans. and Ed.). London: Basic Books. 
Henry, J. D., \& Crawford, J. R. (2004). A meta-analytic review of verbal fluency performance following focal cortical lesions. Neuropsychology, 18(2), 284-295.

Huntley, J. D., \& Howard, R. J. (2010). Working memory in early Alzheimer's disease: A neuropsychological review. International Journal of Geriatric Psychiatry, 25(2), $121-132$.

Insel, T. R. (2010). Rethinking schizophrenia. Nature, 468, 187-193.

Kaplan, H. I., \& Sadock, B. J. (2009). Kaplan \& Sadock's comprehensive textbook of psychiatry. Baltimore, MD: Wolters Kluwer, Lippincott Williams \& Wilkins.

Kay, S. R., Fiszbein, A., \& Opler, L. A. (1987). The positive and negative syndrome scale (PANSS) for schizophrenia. Schizophrenia Bulletin, 13(2), 261-276.

Kotsiantis, S. B. (2007). Supervised machine learning: A review of classification techniques. In I. Maglogiannis, K. Karpouzis, M. Wallace, \& J. Soldatos (Eds.), Emerging artificial intelligence applications in computer engineering: Real world AI systems with applications (pp. 3-24). Amsterdam: IOS Press.

Landauer, T. K., \& Dumais, S. T. (1997). A solution to Plato's problem: The latent semantic analysis theory of acquisition, induction, and representation of knowledge. Psychological Review, 104, 211-240.

Lezak, M. D., Howieson, D. B., Bigler, E. D., \& Tranel, C. (2012). Neuropsychological assessment (5th ed.). New York: Oxford University Press.

Martínez-Sánchez, F., Muela-Martínez, J. A., Cortés-Soto, P., Meilán, J. J. G., Ferrándiz, J. A. V., Caparrós, A. E., \& Valverde, I. M. P. (2015). Can the acoustic analysis of expressive prosody discriminate schizophrenia? The Spanish Journal of Psychology, 18(E86). doi:doi:10.1017/sjp.2015.85.

Montague, P. R., Dolan, R. J., Friston, K. J., \& Dayan, P. (2012). Computational psychiatry. Trends in Cognitive Sciences, 16(1), 72-80.

Mota, N. B., Furtado, R., Maia, P. P., Copelli, M., \& Ribeiro, S. (2014). Graph analysis of dream reports is especially informative about psychosis. Scientific Reports, 4 , 3691. doi:10.1038/srep03691.

Mota, N. B., Vasconcelos, N. A., Lemos, N., Pieretti, A. C., Kinouchi, O., Cecchi, G. A., Copelli, M., \& Ribeiro, S. (2012). Speech graphs provide a quantitative measure of thought disorder in psychosis. PLoS One, 7(4), e34928. doi:10.1371/journal.pone.0034928.

Nickles, L. (2001). Spoken word production. In B. Rapp (Ed.), What deficits reveal about the human mind/brain: A handbook of cognitive neuropsychology: Philadelphia: Psychology Press.

Riedel, W. J. (2014). Preventing cognitive decline in preclinical Alzheimer's disease. Current Opinions in Pharmacology, 14, 18-22.

Scarone, S., Manzone, M. L., Gambini, O., Kantzas, I., Limosani, I., D’Agostino, A., \& Hobson, J. A. (2007). The dream as a model for psychosis: An experimental approach using bizarreness as a cognitive marker. Schizophrenia Bulletin, 34(3), $515-522$.

Stokes, D. E. (Ed.) (1997). Pasteur's quadrant - basic science and technological innovation. Washington, DC: Brookings Institution Press.

Wang, X. J., \& Krystal, J. H. (2014). Computational psychiatry. Neuron, 84(3), 638-654. 